\title{
A manifesto to action: trickle or trailblazing in translational research?
}

\author{
Gabriela Voskerician ${ }^{1}$
}

Published online: 5 October 2015

(c) Springer Science+Business Media New York 2015

Consistently, every year, more than half of the submissions to the Journal of Materials Science: Materials in Medicine flag the "potential" of the discussed innovation for translation into a bedside solution. Yet, even beyond the confines of JMSM, very few groups advance their research beyond a small animal model biocompatibility validation. As a journal and as a research community, we need to look more closely at our capacity to translate discoveries into viable clinical solutions. Where are the barriers and how can they be eliminated?

The Editors would like to encourage an open discussion and assessment of current biomedical research practice. To ensure uniformity, translational research is understood as defined by the National Institutes of Health (NIH), i.e., "the movement of discoveries in basic research to application at the clinical level". Recently I had an opportunity to discuss the state of translational research with Professor James M. Anderson, MD, PhD [Case Western Reserve University, USA], and Professor Yasuhiko Tabata, MD, $\mathrm{PhD}$, D. Pharm. [University of Kyoto, Japan]. Some of their thoughts are incorporated below, and suggest that whether translational research is trickling or reshaping the biomedical research landscape depends on who you ask.

The primary barriers to bedside translation and adoption of biomedical research are often recognized to be: (1) operation within "silos" [no/limited communication/exchange]; (2) research abandoned/stagnant before the biocompatibility validation is completed; (3) operation within a product life-cycle vacuum [no early consideration

Gabriela Voskerician

gxv4@case.edu

1 Department of Biomedical Engineering, Case Western Reserve University, Cleveland, OH, USA towards critical product design parameters for reaching the bedside].

Are we operating under a silo syndrome? The answer seems to be "Yes!". While competition is a healthy practice, restricted or no communication as a result of competition [to publish or attract funding] will impact innovation. We tend to come together under one project goal only when we are forced/enticed by specific funding mechanisms. We challenge our readers to take a hard look at their track record: How many times have you actually pursued a collaboration in the absence of consortium project funding? The traditional scenario plays out as: we come together $\rightarrow$ we put together the consortium $\rightarrow$ the project (unfortunately) does not get funded $\rightarrow$ we say "goodbye" only to return to our own silo. This is the behavior of someone in "survival mode." As scientists, we need funding to survive, hence we try anything and everything, more often than not using a shotgun approach, hoping to find the "right" formula. The danger of the silo effect is highlighted by award-winning writer Gillian Tett's most recent release "The Silo Effect" where she demonstrates the anti-innovation effect brought about by the of absence of collaboration and communication.

Do we have a short attention span, changing research focus gears more often than we should? Again, the answer seems to be "Yes!". How many of us think beyond the 24 well plate biocompatibility validation? How many of us verify the actual potential of the innovation to reach bedside with a colleague clinician beyond a $15 \mathrm{~min}$ phone call or a five sentence e-mail? Preliminary findings are often discarded/abandoned to re-direct the focus towards the post-doc who just brought in some funding tied into a specific research goal. Even more disconcerting is the fact that some of us do not take the time to understand the most appropriate animal model selection and progression [and 
especially limitations] in conducting biocompatibility validations. Just because someone else did it and published it, does not mean that the model was appropriately selected. Do we think for ourselves or do we blindly [while chasing funding] follow because we are too busy to question and ensure reproducibility?

Do we ever seriously think of the practical bedside application? In this case the answer is too often "No!". As research progresses through animal model validation, we should ask ourselves what is the potential of developing an actual product. Industry traditionally calls this perspective the "product life-cycle". This is the time to evaluate the innovation from an enterprise perspective. More often than not, we hear researchers claim that pre-clinical validation is the end of the line for them. Further development would have to follow on someone else's shoulders. While we are not asking the biomedical community to spin-off startups daily, all of us should take an active interest in the fate of what our passion and effort have wrought. Yes, perhaps many academic institutions do not have an enterprise infrastructure. Yes, perhaps dealing with the internal Technology Transfer office bureaucracy is a source of discouragement. Yes, not understanding what it takes to bring a product to market frankly frightens many of us.

However, we have a responsibility to our patients and end-user clinicians and surgeons to bring the innovation to bedside. So what can we do right now?
Two recurring problems are funding and enterprise education. If these can be resolved, theoretically, everything else could beautifully fall into place. The path of least resistance is education and training. Many university centers have open seminars addressing innovation and enterprise. This knowledge could be used to create a personalized seminar series or course module within each Department. The aspect of funding is highly convoluted, involving not just national priorities in funding science, but also ethical concerns in collaborating with non-for-profit organizations. The solution here is simpler than a projected 1000-page volume of policy changes and taxation to increase the grantor agency's budget. Start by evaluating your profile as a researcher, with your unique qualities and limitations, then, pair yourself with a collaborator that complements the strengths while balancing the weaknesses. We offer no guarantees, but your "funding luck" might change. Also, allergies to collaborating with industry do not help, but a healthy evaluation of how a particular entity conducts business does.

I would like to thank Professors Anderson and Tabata for their thoughts and willingness to share their perspectives on the state of translational research. Let us not forget that translational research starts with the patient need and clinician want, not with the small-scale material batch that we just validated at an $n=5$. 